\title{
Performance Evaluation of Wavelength Division Multiplexing Photonic Analogue-to-Digital Converters for High-Resolution Radar Systems
}

\author{
Pedro E. D. Cruz ${ }^{1 *}$, Tiago M. F. Alves ${ }^{1,2}$, Adolfo V. T. Cartaxo ${ }^{1,2}$ \\ ${ }^{1}$ Instituto de Telecomunicações, Lisboa, Portugal \\ ${ }^{2}$ Instituto Universitário de Lisboa (ISCTE-IUL), Lisboa, Portugal \\ Email: *pedro.cruz@lx.it.pt
}

How to cite this paper: Cruz, P.E.D., Alves, T.M.F. and Cartaxo, A.V.T. (2019) Performance Evaluation of Wavelength Division Multiplexing Photonic Analogue-to-Digital Converters for High-Resolution Radar Systems. Optics and Photonics Journal, 9, 219-234.

https://doi.org/10.4236/opj.2019.912018

Received: December 22, 2019

Accepted: January 13, 2020

Published: January 16, 2020

Copyright $\odot 2019$ by author(s) and Scientific Research Publishing Inc. This work is licensed under the Creative Commons Attribution International License (CC BY 4.0).

http://creativecommons.org/licenses/by/4.0/

\begin{abstract}
The performance of the wavelength division multiplexing (WDM) photonic analogue-to-digital converter (ADC) used for digitization of high-resolution radar systems is evaluated numerically by using the peak signal-to-noise ratio (SNR) metric. Two different WDM photonic ADC architectures are considered for the digitization of radar signals with $5 \mathrm{GHz}$ of bandwidth (spatial resolution of $3 \mathrm{~cm}$ ), in order to provide a comprehensive study of the compromises present when deploying radar signals with high-resolution: 1) a four-channel architecture with each channel employing an ADC with 5 GSamples/s, and 2) an eight-channel architecture with each channel employing an ADC with $2.5 \mathrm{GSamples/s.} \mathrm{For} \mathrm{peak} \mathrm{powers} \mathrm{of} \mathrm{the} \mathrm{pulsed} \mathrm{source} \mathrm{be-}$ tween 10 and $20 \mathrm{dBm}$ and a distance between the radar antenna and the sensing object of 2.4 meters, peak SNR levels between 29 and $39 \mathrm{~dB}$ are achieved with the eight-channel architecture, which shows higher peak SNR levels when compared with the four-channel architecture. For the eight-channel architecture and for the same peak powers of the pulsed source, peak SNR levels between 11 and $16 \mathrm{~dB}$ are obtained when the distance increases to 13.5 meters. With this evaluation using the peak SNR, it is possible to assess the performance limits when choosing a specific radar range, while keeping the same resolution.
\end{abstract}

\section{Keywords}

Analogue-to-Digital Converter, Radar, Signal-to-Noise Ratio, Wavelength Division Multiplexing 


\section{Introduction}

Modern radar applications, such as driverless automobiles and unmanned aerial vehicles [1], require high-range, high sampling speed and high-resolution [2] [3] [4]. To achieve high-resolution in radar systems, a radar signal with large bandwidth is required [1]. However, the digitization of a high-bandwidth radar signal requires analogue-to-digital converters (ADCs) with high-speed and large-bandwidth, which are limited in terms of resolution and electronic timing jitter [5]. Thus, ADC operation is key in the radar performance [6].

Recently, radar systems have benefited considerably from the development of microwave photonic technology, and the existence of mode-locked lasers generating ultra-short pulses with reduced aperture jitter has opened the door for photonic ADCs [6] [7] [8] [9] [10]. A photonic ADC employs an ultra-stable mode-locked laser that provides an array of ultra-short optical pulses which define the sampling instants for the signal to be digitized [11]. At the output of the mode-locked laser, a high-speed electro-optic modulator samples the signal of interest, at the pace of the mode-locked laser [8] [11]. The optical signal containing the information of the signal of interest as an amplitude variation is then converted to electrical using a high-speed photodiode and quantized by an electronic ADC. This procedure ensures that all the timing issues are controlled by the mode-locked laser. This includes the sampling rate, which is controlled by the pulse repetition rate of the laser, and the timing jitter, which is ruled by the laser jitter [8] [11]. This solution does not reduce the rate of the electronic $\mathrm{ADC}$ as a single $\mathrm{ADC}$ is still responsible for the quantization of the signal at the photodiode output. An effective way to overcome this bottleneck is to employ a time-wavelength interleaving scheme [6] [7] that can relax the front-end bandwidth requirements. For example, a high-bandwidth photodiode and a high-sampling rate $\mathrm{ADC}$ can be replaced into a structure with different channels where each channel employs a lower-bandwidth and low-cost photodiode and an off-the-shelf low-speed ADC, removing the impact of comparator ambiguity which occurs with high-speed ADCs [5] [8] [11]. For instance, a single ADC with sampling rate of $20 \mathrm{GSamples/s} \mathrm{can} \mathrm{be} \mathrm{replaced} \mathrm{by} \mathrm{a} \mathrm{time-wavelength} \mathrm{interleaved} \mathrm{photon-}$ ic ADC with 4 channels, where each channel employs an ADC with 5 GSamples/s. With a time-wavelength interleaved photonic ADC, the mode-locked laser, or pulsed source, which generates a signal with ultra-short pulses with a given repetition rate, is sliced in frequency into $N$ different channels, forming narrower-band pulsed signals at different wavelengths. Adequate time-separation between the pulsed signals is then guaranteed using tunable delay lines, to form a time-wavelength interleaved sampling clock with $N$ times greater repetition rate than the one of the original pulsed source. A high-speed electro-optic modulator is then used to modulate the RF signal onto the interleaved sampling clock. At the receiver side, the optical signal is demultiplexed into $N$ channels, which are converted to the electrical domain using low-cost photodiodes. The electrical signals of the $N$ channels are digitized by $N$ low-sampling rate ADCs 
and the $N$ digitized signals are combined using digital-signal processing to form the digitized signal.

So far, the capabilities of time-wavelength interleaved photonic ADCs have been tested mainly using single-tone signals as RF inputs [2] [6] [7] [8] [12]. However, in practical radar systems, wideband input signals are normally used [13]. In [13] and [14], a wideband radar signal with a frequency range of $8-12$ $\mathrm{GHz}$ is digitized using a time-wavelength interleaved photonic ADC. In this work, the time-wavelength interleaved photonic ADC is used to digitize a radar signal with large bandwidth $(5 \mathrm{GHz})$ and small time width (200 ps) as RF input, which provides high-resolution ( 3 centimeters). The performance of the photonic ADC in this work is evaluated for different architectures and distances between the radar antenna and the sensing object in order to provide a comprehensive study of the compromises present when deploying radar signals with high-resolution. Additionally, the time-stretching operation is employed, which compresses the bandwidth of each channel, relaxing the front-end requirements [10] [15]. Time-stretching is achieved using two dispersive elements, one before optical modulation, to produce a chirped pulsed source, and another dispersive element (with higher length than the first dispersive element) to time-stretch the signal before conversion to the electrical domain [10].

When digitizing radar signals with photonic ADCs, two of the main constraints are the very low signal power captured by the radar antenna, and the signal losses coming from its photonic structure. Therefore, electrical and optical amplifiers with large gain are required, leading to signal-to-noise ratio (SNR) degradation [15]. In this work, the performance of the time-wavelength interleaved photonic $\mathrm{ADC}$, hereafter referred as wavelength division multiplexing (WDM) photonic $(\mathrm{Ph})-\mathrm{ADC}$, is assessed by investigating the compromise between the peak SNR of the recovered samples of the radar signal and the distance between the radar antenna and the sensing object, for optical pulsed sources with different peak powers and WDM Ph-ADCs with different number of channels. With this evaluation, it is possible to assess the performance limits when choosing a specific radar range, while keeping the same resolution.

The paper is structured as follows. Section 2 presents and explains the WDM photonic ADC architecture and its parameters. The WDM photonic ADC architecture enables relaxing the sampling rate of ADCs required to digitize the radar signals employed in systems with high spatial resolution. Section 3 presents the performance results (obtained with the SNR metric) of the recovered samples of the radar signal when using the WDM photonic ADC architecture. The performance results are evaluated and discussed for two different architectures: 1) a four-channel architecture with each channel employing an ADC with 5 GSamples/s, and 2) an eight-channel architecture with each channel employing an ADC with 2.5 GSamples/s. Section 4 summarizes the main conclusion.

\section{WDM Photonic ADC}

In this section, the WDM Ph-ADC architecture is presented, and the parameters 
of the different Ph-ADC components and compromises between them are explained.

\subsection{WDM Photonic ADC Architecture}

The WDM Ph-ADC architecture is described in this subsection. Figure 1(a) shows the WDM Ph-ADC architecture. Figure 1(b) illustrates the waveforms at different points of the WDM Ph-ADC architecture, for an example where three samples inside the radar signal are recovered.

The pulsed source generates a signal with ultra-short non-chirped Gaussian pulses with peak power $P_{\mathrm{p}}$, repetition period $T_{\text {rep }}$ and repetition rate $f_{\text {rep }}\left(f_{\text {rep }}=\right.$ $\left.1 / T_{\text {rep }}\right)$. The wideband spectrum of the pulsed source is sliced by a 1 -to- $N$ arrayed waveguide grating (AWG1) which forms narrower band pulsed signals at different wavelengths. $N$ corresponds to the number of channels of the WDM

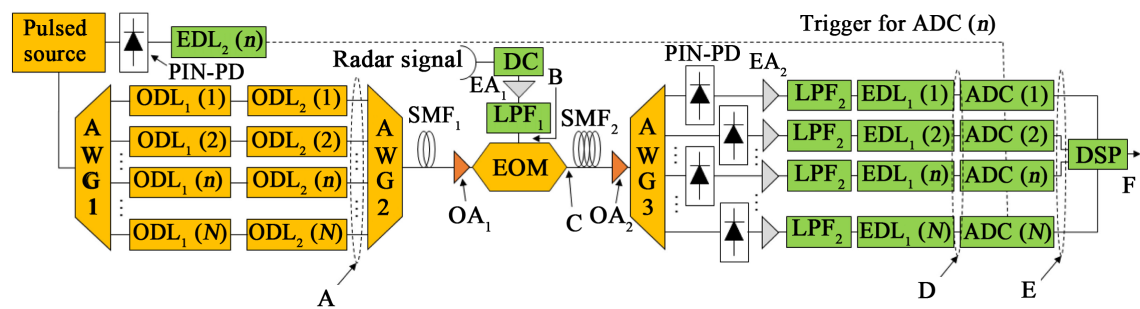

(a)
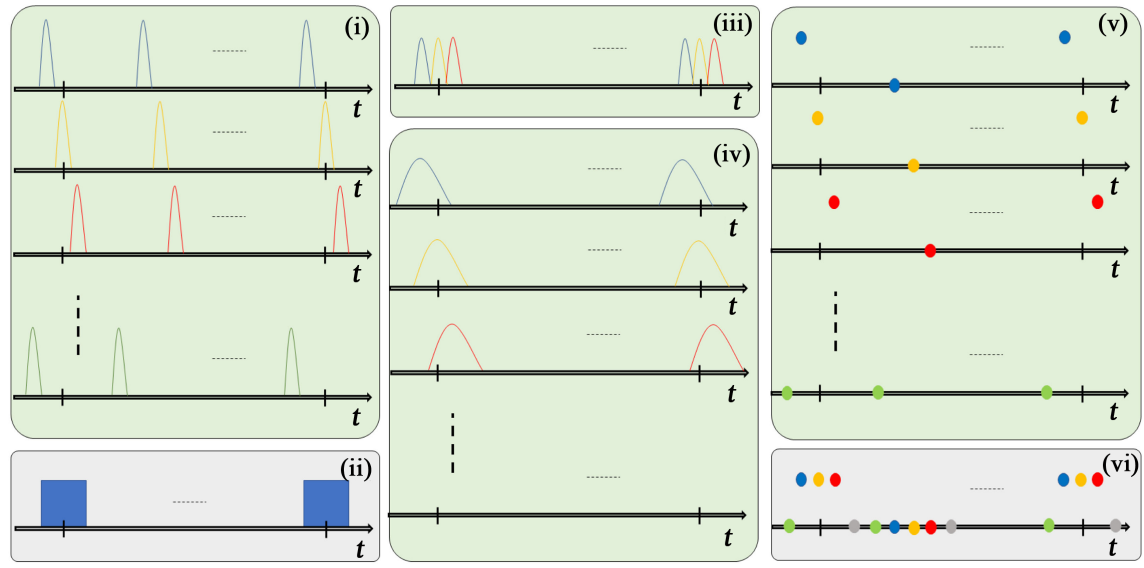

(b)

Figure 1. (a) WDM Ph-ADC architecture and (b) illustrative figures of the waveforms at different points of the WDM Ph-ADC architecture, for an example where three samples inside the radar signal are recovered (corresponding to the channels which have optical pulsed sequences with blue, yellow and red colors). In (a): AWG-arrayed waveguide grating; DC-down-converter; DSP_digital signal processing; EA-electrical amplifier; EDL-electrical delay line; EOM-electro-optic modulator; LPF-low-pass filter; $\mathrm{OA}$-optical amplifier; ODL_optical delay line; PIN-PD_PIN photodiode; SMF—single mode fibre. In (b): (i) optical pulsed sequences prior to electro-optic modulation (point A in (a)); (ii) radar signal (point B in (a)); (iii) modulated optical pulsed sequences (point C in (a)); (iv) time stretched pulsed sequences before digitization (point D in (a)); (v) digitized waveforms after the ADCs (point E in (a)); (vi) reconstructed digitized radar waveform (point $\mathrm{F}$ in (a)). 
$\mathrm{Ph}$-ADC. In the illustration of Figure $1(\mathrm{~b})$ it is assumed that the wideband spectrum of the pulsed source is much greater than the bandwidth of the WDM signal to ensure that the different wavelengths have similar power. An optical delay line $\left(\mathrm{ODL}_{1}\right)$ is introduced to ensure adequate time-separation between the pulsed signals of each WDM channel in order to sample the radar signal sequentially over time. The time delay from $\mathrm{ODL}_{1}$ is given by $\operatorname{ODL}_{1}(n)=n \cdot \Delta_{\text {res }}$, where $\Delta_{\text {res }}=T_{\text {rep }} / N$ is the time resolution of the digitized radar waveform, and $n$ is the channel index $(n=1,2, \cdots, N)$. $\mathrm{ODL}_{2}$ is used to compensate for the walk-off effect suffered by each WDM channel due to the propagation over the first single mode fiber (SMF) span $\left(\mathrm{SMF}_{1}\right.$ with length $\left.L_{1}\right)$ [3] (point A in Figure 1 (a) and subfigure (1) in Figure $1(\mathrm{~b}))$. The time delays introduced by $\mathrm{ODL}_{2}(n)$ are equal to $K(n) \cdot D_{\lambda} \cdot L_{1} \cdot \Delta \lambda$, with $K(n)=-[(N+1) / 2]+n$ for $N$ odd, and $K(n)=-[(N / 2)+1]+n$ for $N$ even, where $D_{\lambda}$ is the fibre dispersion parameter in $\mathrm{ps} / \mathrm{nm} / \mathrm{km}, L_{1}$ is the first span length in $\mathrm{km}$, and $\Delta \lambda$ is the AWG channel spacing in nm. AWG2 combines the pulsed signals of the different channels in a pulsed signal with consecutive pulses separated in wavelength. The signal at the AWG2 output is propagated along the $\mathrm{SMF}_{1}$ span, which broadens the optical pulses and introduces walk-off between them (previously compensated by $\mathrm{ODL}_{2}$ ) [3]. The signal after propagation along $\mathrm{SMF}_{1}$ is a pulsed signal with a repetition period given by $\Delta_{\text {res }}$. Afterwards, amplification is performed by an optical amplifier $\left(\mathrm{OA}_{1}\right)$ to compensate for the accumulated losses of the AWGs and the first SMF span. In the estimation of the noise introduced by the optical amplifiers, it is assumed that the amplified spontaneous emission (ASE) noise is modelled by a zero mean Gaussian noise with power spectral density (PSD) along one polarization direction (parallel or perpendicular) and along one signal component (in-phase or quadrature) given by $S_{\mathrm{o} 1}=(1 / 4) \cdot h \cdot v \cdot f_{\mathrm{n}} \cdot\left(g_{01}-1\right)$, where $h$ is the Planck constant, $v$ is the central frequency of the optical signal spectrum, $f_{\mathrm{n}}$ is the $\mathrm{OA}_{1}$ noise figure and $g_{\mathrm{ol}}$ is the $\mathrm{OA}_{1}$ gain.

The signal at the $\mathrm{OA}_{1}$ output feeds the electro-optic modulator (EOM), which modulates the optical pulses by the high-resolution radar signal to be sampled (point C in Figure 1(a) and subfigure (3) in Figure 1(b)). The EOM is described by a linear input-output characteristic. This model is valid when the modulation depth of the electrical signal driving the EOM is sufficiently small to ensure linear operation. For spatial resolutions of the order of a few centimetres, radar bandwidths of the order of a few $\mathrm{GHz}$ are required. The spatial resolution of a radar signal, $\Delta d$, is related with the radar bandwidth $B_{\mathrm{r}}$. This relation is given by $\Delta d=c /\left(2 \cdot B_{\mathrm{r}}\right)[16]$, where $c$ is the speed of light in a vacuum. For instance, a radar signal with a $5 \mathrm{GHz}$ bandwidth leads to a spatial resolution of $3 \mathrm{~cm} \mathrm{[3].}$ The number of samples along the radar pulse width, $N_{\mathrm{s}}$, is related with $\Delta_{\text {res }}$ by

$$
N_{\mathrm{s}}=\left\lfloor 1 /\left(B_{\mathrm{r}} \cdot \Delta_{\text {res }}\right)\right\rfloor=\left\lfloor N /\left(B_{\mathrm{r}} \cdot T_{\text {rep }}\right)\right\rfloor
$$

where $\lfloor\cdot\rfloor$ corresponds to the operation of approximation to the nearest lowest integer. The radar signal is emitted by the radar antenna in order to "sense" the distance of the objects in the surrounding environment (few meters range). The 
signal loss of the path radar-object-radar is described by the free-space path loss (FSPL), which is given by:

$$
\operatorname{FSPL}[\mathrm{dB}]=P_{\text {out }}-P_{\text {in }}=20 \cdot \log _{10}\left[\left(4 \cdot \pi \cdot 2 \cdot d \cdot f_{0}\right) / c\right]
$$

where $P_{\text {out }}$ is the output power of the radar signal sent by the antenna in $\mathrm{dBm}, P_{\text {in }}$ is the radar received power in $\mathrm{dBm}, d$ is the distance between the antenna and the sensing object and $f_{0}$ is the central frequency of the radar signal. The received radar signal (with average power $P_{\text {in }}$ ) is then amplified by an electrical amplifier $\left(\mathrm{EA}_{1}\right)$ and filtered by a Gaussian-shape low-pass filter $\left(\mathrm{LPF}_{1}\right)$ (point $\mathrm{B}$ in Figure 1(a) and subfigure (2) in Figure $1(\mathrm{~b})$ ). The $\mathrm{EA}_{1}$-generated electrical noise is modelled as zero mean additive Gaussian noise with a two-sided PSD given by

$$
S_{\mathrm{e}}(f)=2 \cdot k_{\mathrm{B}} \cdot T \cdot R_{\mathrm{L}} \cdot f_{\mathrm{e}} \cdot g_{\mathrm{e}}^{2}
$$

where $k_{\mathrm{B}}$ is the Boltzmann constant $\left(k_{\mathrm{B}}=1.38 \times 10^{-23} \mathrm{~J} \cdot \mathrm{K}^{-1}\right), T$ is the temperature in Kelvin, $R_{\mathrm{L}}$ is the load resistance, $f_{\mathrm{e}}$ is the $\mathrm{EA}_{1}$ noise figure and $g_{\mathrm{e}}$ is the voltage gain of the electrical amplifier $\mathrm{EA}_{1}$.

After electro-optic modulation, a second SMF span $\left(\mathrm{SMF}_{2}\right)$, with the same dispersion parameter as $\mathrm{SMF}_{1}$ but with length $L_{2}\left(L_{2}>L_{1}\right)$, broadens even more the optical pulses causing time-stretching [15]. The time-stretching operation compresses the spectrum at the receiver-side and is regulated by the stretching factor $M=1+\left(L_{2} / L_{1}\right)$ [15]. Then, the optical signal is amplified by the optical amplifier $\mathrm{OA}_{2}$, which compensates for the EOM losses and the losses of the second SMF span. The optical signal at the $\mathrm{OA}_{2}$ output is demultiplexed by AWG3 to photodetect individually each channel. For the photodetection operation, PIN-photodetectors (PDs) are employed to convert the intensity fluctuations of the optical pulses to electrical current. The photodetected signals of each channel are then amplified ( $\mathrm{EA}_{2}$ amplifiers) and filtered by a Gaussian-shape low-pass filter $\left(\mathrm{LPF}_{2}\right.$ filters) with bandwidth equal to half of the repetition rate of the pulsed source ( $\mathrm{LPF}_{2}$ emulates the bandwidth of each $\mathrm{ADC}$, which should be half of the $\mathrm{ADC}$ sampling rate). The $\mathrm{EA}_{2}$-generated current noise is modelled as an additive Gaussian thermal noise with two-sided PSD given by

$$
S_{r}(f)=2 \cdot k_{\mathrm{B}} \cdot T \cdot f_{\mathrm{r}} \cdot g_{\mathrm{r}}^{2} / R_{\mathrm{L}}
$$

where $f_{\mathrm{r}}$ is the $\mathrm{EA}_{2}$ noise figure and $g_{\mathrm{r}}$ is the voltage gain of the electrical amplifier $\mathrm{EA}_{2}$. An electrical delay line $\left(\mathrm{EDL}_{1}\right)$ is introduced to compensate for the walk-off effect suffered by each WDM channel due to the propagation over the second SMF span [3]. $\operatorname{EDL}_{1}(n)$ adds time delays equal to $K(n) \cdot D_{\lambda} \cdot L_{2} \cdot \Delta \lambda$. The delay-compensated signals of each channel are fed to the ADCs (point D in Figure 1(a) and subfigure (4) in Figure 1(b)), which are responsible for the digitization of the waveforms of each channel (point E in Figure 1(a) and subfigure (5) in Figure 1(b)). These ADCs have to be adequately synchronized by a trigger signal in order to sample the pulsed signals in the peak of each pulse [3]. The trigger associated with the sampling process of each ADC is obtained directly from the pulsed source signal, which has been previously photodetected. To en- 
sure that the trigger, for each ADC, matches precisely the time instants corresponding to the maximum of the optical pulses, a time delay is introduced after the photodetection of the original pulsed signal. This delay is provided by $\mathrm{EDL}_{2}(n)$, which is equal to $\mathrm{ODL}_{1}(n): \mathrm{EDL}_{2}(n)=\mathrm{ODL}_{1}(n)=n \cdot \Delta_{\text {res }}$. The digitized waveform is then obtained in the digital signal processing (DSP) block (point F in Figure 1(a) and subfigure (6) in Figure 1(b)), that receives and combines the digital signals of the different ADCs.

\subsection{WDM Photonic ADC Parameters}

Different combinations of number of channels and repetition rates of the pulsed source can be considered. These combinations are ruled by the relation between $N$ and $f_{\text {rep. }}$. If the number of samples along the radar pulse width and the bandwidth of the radar signal are maintained, a decrease by half of the repetition rate of the pulsed source implies twice the number of channels. Using a pulsed source with half of the repetition rate implies ADCs at the receiver front-end with half of the sampling rate, which is less cost-demanding. If the main goal is to reduce the number of channels, ADCs with higher sampling rate are required. Therefore, a compromise between the number of channels and repetition rate of the pulsed source dictates the structure of the WDM Ph-ADC. With $f_{\text {rep }}=5 \mathrm{GHz}$ and $N=4$, the Ph-ADC architecture employs ADCs with 5 GSamples/s, and the number of channels is not considerably high. If ADCs with lower sampling rate are required, a solution with $f_{\text {rep }}=2.5 \mathrm{GHz}$ can be employed, which leads to an architecture with 8 channels. Another possibility is to reduce the number of channels, at the expense of increasing the repetition rate of the pulsed source and, in the same way, the ADC sampling rate. For instance, $f_{\text {rep }}=10 \mathrm{GHz}$ results in a WDM Ph-ADC architecture with 2 channels and employing ADCs with 10 GSamples/s.

In this work, a radar bandwidth of $5 \mathrm{GHz}$ is considered, which leads to a radar pulse width (equal to $1 / B_{r}$ ) of 200 ps. Additionally, it is assumed that $\Delta_{\text {res }}=50 \mathrm{ps}$, which indicates that 4 samples along the radar pulse width of 200 ps can be recovered. With 4 channels, it is possible to recover one sample of the radar signal per channel. With 8 channels, there will be channels which will not capture samples inside the radar signal when $\Delta_{\text {res }}=50$ ps. If the number of channels is reduced to 2, each channel is responsible for recovering 2 samples of the radar signal. In this work, two different WDM $\mathrm{Ph}-\mathrm{ADC}$ architectures are considered: 1) architecture A with $f_{\text {rep }}=5 \mathrm{GHz}$ and $N=4$, which represents a good compromise between number of channels and ADC sampling rate, and 2) architecture $B$ with $f_{\text {rep }}=2.5 \mathrm{GHz}$ and $N=8$, which is less demanding in terms of ADC sampling rate but requires a higher number of WDM channels.

The WDM Ph-ADC employs a pulsed source with $5 \mathrm{GHz}$ and $2.5 \mathrm{GHz}$ repetition rate for architectures $A$ and $B$, respectively, and the pulsed source has a -3 $\mathrm{dB}$ bandwidth of $2 \mathrm{~nm}$, similar to the ones shown in [17] [18]. Each AWG has an insertion loss of $5 \mathrm{~dB}$, a frequency response modelled by a Gaussian filter with $-3 \mathrm{~dB}$ bandwidth of $0.2 \mathrm{~nm}$, and the channels are separated by $0.5 \mathrm{~nm}$ and 0.25 
nm for architectures A and B, respectively. This channel separation ensures that the power loss suffered by the edge channels is similar for the two architectures. The ODL cascade $\left(\mathrm{ODL}_{1}\right.$ and $\left.\mathrm{ODL}_{2}\right)$ insertion losses are $1 \mathrm{~dB}$. The $\mathrm{SMF}$ has a dispersion parameter of $17 \mathrm{ps} / \mathrm{nm} / \mathrm{km}$ (at the operating wavelength of $\lambda_{0}=1550$ $\mathrm{nm}$ ) and attenuation coefficient of $0.2 \mathrm{~dB} / \mathrm{km}$. The following values are used to estimate the $\mathrm{OA}_{1}$ noise PSD: $h=6.626 \times 10^{-34} \mathrm{~J} \cdot \mathrm{s}, v=193.1 \mathrm{THz}$ and $f_{\mathrm{n}}=4 \mathrm{~dB}$. It is assumed that the insertion loss of the EOM is $6 \mathrm{~dB}$.

The following values are considered in the estimation of the free-space path loss: $P_{\text {out }}=20 \mathrm{dBm}$ (effective isotropic radiated power) and $f_{0}=28 \mathrm{GHz}$. The $\mathrm{LPF}_{1}$ filter has a $-3 \mathrm{~dB}$ bandwidth of $10 \mathrm{GHz}$. The following parameters are considered in the estimation of the EA $\mathrm{E}_{1}$ noise PSD: $T=290 \mathrm{~K}, R_{\mathrm{L}}=50 \Omega$ and $f_{\mathrm{e}}=4$ $\mathrm{dB}$. The stretching factor $M$ is 10 , which is achieved by having $\mathrm{SMF}_{1}$ with a length of 500 meters and $\mathrm{SMF}_{2}$ with $4.5 \mathrm{~km}$-length. For the photodetection operation, PIN-photodetectors (PDs) with non-limiting band and responsivity of $0.8 \mathrm{~A} / \mathrm{W}$ are employed. The noise figure of the $\mathrm{EA}_{2}$ amplifier employed after each PIN-PD $\left(f_{\mathrm{r}}\right)$ is $6 \mathrm{~dB}$. The ADCs have $-3 \mathrm{~dB}$ bandwidth equal to half of the repetition rate of the pulsed source $(2.5 \mathrm{GHz}$ for architecture $\mathrm{A}$ and $1.25 \mathrm{GHz}$ for architecture $\mathrm{B}$ ) and are modelled by the $\mathrm{LPF}_{2}$ filters.

\section{Methods, Performance Results and Discussion}

In this section, the performance of the WDM Ph-ADCs used to digitize the high-resolution radar signal is evaluated using numerical simulation in MATLAB. The SNR is considered as a metric for performance evaluation and is estimated using Monte Carlo simulation. The noise sources described in Section 2 are simulated using Gaussian distributed random numbers with appropriate variances. The radar waveform is generated in a time window with duration of 200 ns, comprising 2 radar pulses with a period of 100 ns. The results consider: 1) FSPL $\in\{75,80,85,90\} \mathrm{dB} ; 2)$ peak power of the pulsed source $\left(P_{\mathrm{p}}\right) \in\{10,15,20\}$ $\mathrm{dBm}$; 3) SNR estimates evaluated over 300 signal runs (difference between SNR levels with 300 runs and 1000 runs does not exceed $0.2 \mathrm{~dB}$ ); and 4) the electrical and optical amplifiers $\left(\mathrm{EA}_{1}\right.$ with gain $G_{\mathrm{e}}, \mathrm{EA}_{2}$ with gain $G_{\mathrm{r}}, \mathrm{OA}_{1}$ with gain $G_{\mathrm{o}}$ and $\mathrm{OA}_{2}$ with gain $G_{\mathrm{o} 2}$ ) with gains $\in\{20,30,40\}$ dB. For practical reasons, combinations of the $\mathrm{Ph}-\mathrm{ADC}$ parameters leading to power levels at the $\mathrm{OA}$ input higher than $0 \mathrm{dBm}$ are excluded. The maximum output power of the $\mathrm{OAs}$ is also set to $20 \mathrm{dBm}$. Additionally, for each PIN-PD, the input power should be between $-15 \mathrm{dBm}$ and $0 \mathrm{dBm}$ in order to avoid damaging and ensure the signal power is sufficient to be detected by the PIN-PD.

Table 1 shows the radar received power and the distance between the radar antenna and the sensing object as a function of the FSPL. The analysed configurations correspond to radar-object distances that do not exceed 13.5 meters.

Figure 2 shows the optical spectra of the pulsed source and of the channels at the AWG1 output, for architectures A and B, respectively. Figure 2 shows that, due to the power attenuation induced by the non-ideal amplitude response of 


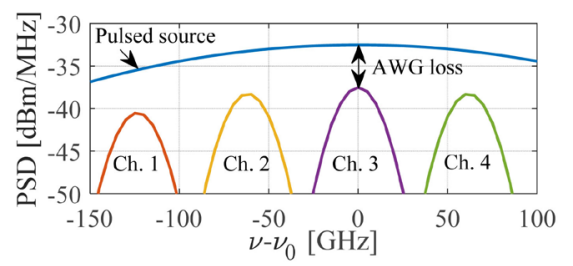

(a)

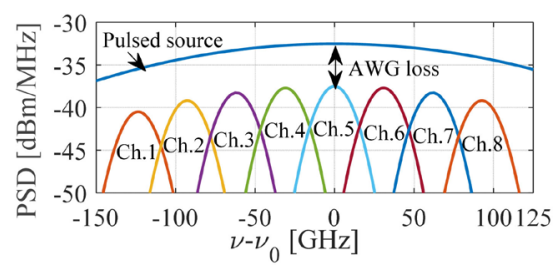

(b)

Figure 2. Optical spectra of the pulsed source and of the channels at the AWG1 output, for (a) architecture A (4 channels) and (b) architecture B (8 channels), with $P_{\mathrm{p}}=15 \mathrm{dBm}$.

Table 1. Radar received power $\left(P_{\text {in }}\right)$ and distance between the radar antenna and the sensing object for different FSPLs.

\begin{tabular}{ccc}
\hline FSPL $[\mathrm{dB}]$ & $P_{\text {in }}[\mathrm{dBm}]$ & Distance $[\mathrm{m}]$ \\
\hline 75 & -55 & 2.4 \\
80 & -60 & 4.3 \\
85 & -65 & 7.6 \\
90 & -70 & 13.5 \\
\hline
\end{tabular}

the pulsed source, the pulses of the different channels have different peak powers. The peak power difference does not exceed $3 \mathrm{~dB}$ among all channels, for both architectures. The power attenuation between different channels can be decreased whether by having a pulsed source with higher bandwidth or by decreasing the AWG channel spacing. However, the AWG channel spacing can only be reduced to a certain level, to avoid significant interference between the different channels.

Figure 3 shows a portion of the signal power at the output of the first SMF span, for architectures A and B, respectively. This signal is periodic in time with one period represented between 48.9 and $49.1 \mathrm{~ns}$ for architecture $\mathrm{A}$, and between 48.8 and $49.2 \mathrm{~ns}$ for architecture B. The pulses with the lowest and highest amplitudes correspond to the ones generated by channel 1 and channel 3 for architecture A, and by channel 1 and channel 5 for architecture B. Figure 3 also shows that the power level is relatively low for both architectures. To compensate this, optical amplification is employed before electro-optic modulation.

Figure 4 shows the radar pulse before and after low-pass filtering with a $\mathrm{LPF}_{1}$ filter with $-3 \mathrm{~dB}$ bandwidth of $10 \mathrm{GHz}$ (the radar signal is null outside the interval shown in Figure 4). The radar pulse is centered at $49 \mathrm{~ns}$ and its width is 200 ps (between 48.9 and $49.1 \mathrm{~ns}$ ).

Figure 5 shows the signal power at the EOM output, for both architectures. Figure 5 shows that, for architectures $\mathrm{A}$ and $\mathrm{B}$, the pulses corresponding to the different channels have different powers. This difference is attributed to the non-flat amplitude response of the pulsed source and to the non-flat amplitude of the radar pulse at the EOM input (as shown in Figure 4 by the continuous line). Figure 5 also shows that, when comparing the powers of both architectures, the powers of architecture A have a higher difference. This is attributed to 


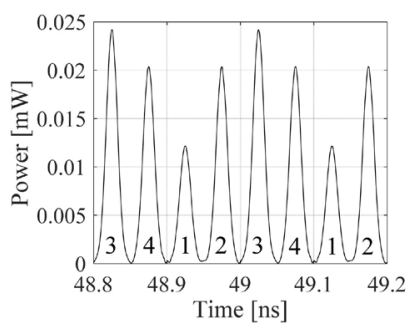

(a)

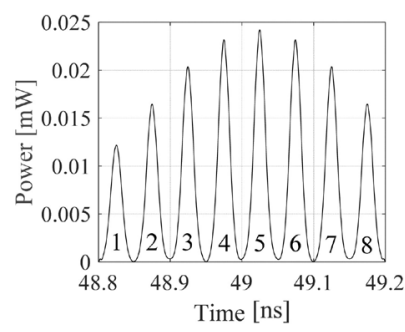

(b)

Figure 3. Portion of the signal power at the output of the first SMF span $\left(P_{\mathrm{p}}=15 \mathrm{dBm}\right)$, for (a) architecture A and (b) architecture B. The channel index corresponding to each pulse is also shown.

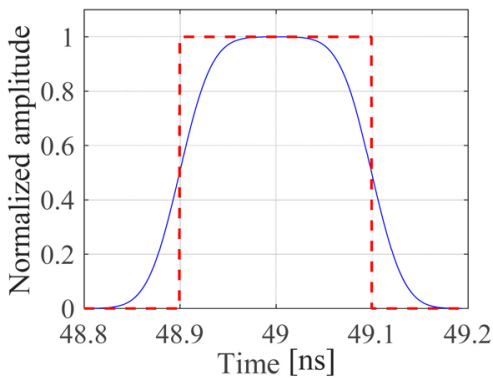

Figure 4. Time waveform of the radar pulse before (dashed line) and after (continuous line) low-pass filtering with a $\mathrm{LPF}_{1}$ filter with $-3 \mathrm{~dB}$ bandwidth of $10 \mathrm{GHz}$.

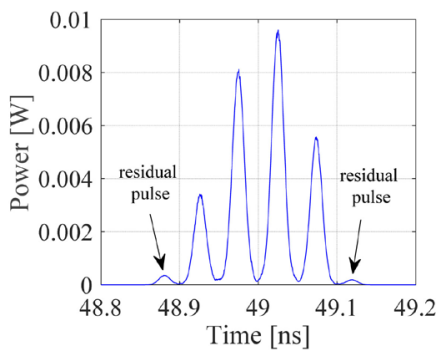

(a)

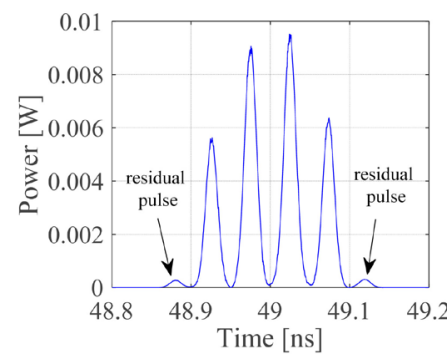

(b)

Figure 5. Signal power at the EOM output $\left(P_{\mathrm{p}}=15 \mathrm{dBm}, G_{\mathrm{e}}=40 \mathrm{~dB}, G_{\mathrm{o} 1}=40 \mathrm{~dB}\right.$, and $P_{\text {in }}$ $=-65 \mathrm{dBm})$, for (a) architecture $\mathrm{A}$ and (b) architecture $\mathrm{B}$.

the channel spacing, which is smaller in architecture B. This translates into less power attenuation between consecutive channels for architecture B, when comparing with architecture A. Each pulse inside the radar pulse width (between 48.9 and $49.1 \mathrm{~ns}$ ) in architecture A corresponds to a different channel of the total of 4 channels. For architecture A, the power difference between channels 1 (most-affected by the non-flat amplitude response of the pulsed source) and 3 (least-affected by the non-flat amplitude response of the pulsed source) is $3 \mathrm{~dB}$. For architecture B, the pulses inside the radar pulse width (between 48.9 and $49.1 \mathrm{~ns}$ ) correspond to the channels with indexes 3, 4, 5 and 6 (channels 1, 2, 7 and 8 do not capture samples inside the radar pulse width). For architecture B, the power difference between channels 3 (channel that captures a sample inside the radar pulse width and is most-affected by the non-flat amplitude response of 
the pulsed source) and 5 (channel that captures a sample inside the radar pulse width and is least-affected by the non-flat amplitude response of the pulsed source) is $0.75 \mathrm{~dB}$.

Figure 6 shows the signal voltages at the ADC input, for different channels of both architectures. It shows clearly the time-spreading, together with the difference in amplitude between different channels, also attributed to the non-flat amplitude response of the pulsed source and the non-flat amplitude of the radar pulse at the EOM input. Figure 6 also shows that the spreading in time is stronger for architecture $\mathrm{B}$. This is a consequence of the bandwidth of the low-pass filter $\mathrm{LPF}_{2}$, which is lower for architecture $\mathrm{B}$.

The power difference verified for different channels induces different performance among the WDM Ph-ADC channels. This performance can be quantified using the SNR per channel. The SNR is given by the ratio between the instantaneous signal power and the variance of the noise introduced by the Ph-ADC. When recovering the radar samples at the receiver side, we are most interested in the peak SNR (average power ratio between the peak of the instantaneous signal power and the noise variance at the same time instant), which gives the best performance level when recovering the radar samples [15]. For this reason, the SNR levels shown in this section correspond to the peak SNR levels.

Figure 7 shows the instantaneous signal power and the noise variance of the different channels of architecture A, at each $\mathrm{LPF}_{2}$ output, for $P_{\mathrm{p}}=15 \mathrm{dBm}, G_{\mathrm{e}}=$ $40 \mathrm{~dB}, G_{\mathrm{o} 1}=40 \mathrm{~dB}, G_{\mathrm{o} 2}=30 \mathrm{~dB}, G_{\mathrm{r}}=20 \mathrm{~dB}$ and $P_{\mathrm{in}}=-65 \mathrm{dBm}$. Figure 7 shows that a residual pulse appears in the signals of channel 1 and channel 4 . These residual pulses are the ones shown in Figure 5(a), which result from the non-ideal amplitude response of the radar pulse at the EOM input. The apparent similarity of the pulses amplitudes in Figure 7 results from the fact that the vertical scale in Figure 7 is a decibel scale. Figure 7 also shows that most of the signal power and noise variance are confined in a time slot similar to the radar pulse width.

Figure 8 shows the instantaneous signal power and the noise variance of the different channels of architecture B, at each $\mathrm{LPF}_{2}$ output, for $P_{\mathrm{p}}=15 \mathrm{dBm}, G_{\mathrm{e}}=$ $40 \mathrm{~dB}, G_{\mathrm{o} 1}=40 \mathrm{~dB}, G_{\mathrm{o} 2}=30 \mathrm{~dB}, G_{\mathrm{r}}=20 \mathrm{~dB}$ and $P_{\mathrm{in}}=-65 \mathrm{dBm}$. Figure 8 shows

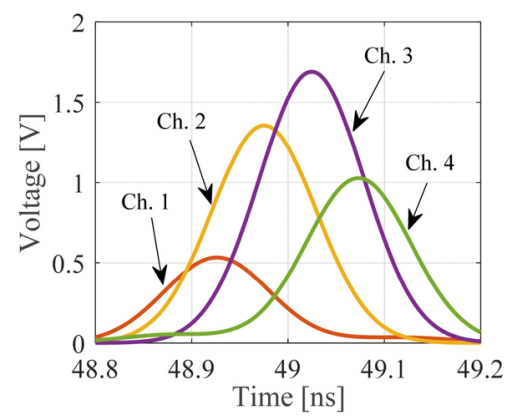

(a)

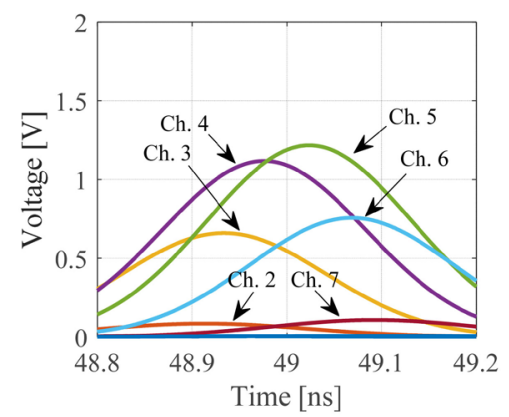

(b)

Figure 6. Signal voltage at the ADC input for different channels $\left(P_{\mathrm{p}}=15 \mathrm{dBm}, G_{\mathrm{e}}=40\right.$ $\mathrm{dB}, G_{\mathrm{o} 1}=40 \mathrm{~dB}, G_{\mathrm{o} 2}=30 \mathrm{~dB}, G_{\mathrm{r}}=20 \mathrm{~dB}$ and $P_{\mathrm{in}}=-65 \mathrm{dBm}$ ), for (a) architecture A and (b) architecture B. 

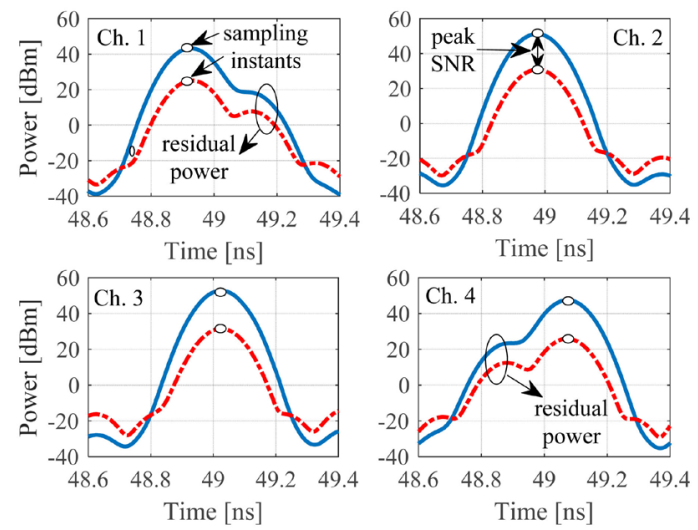

Figure 7. Instantaneous signal power (continuous line) and noise variance (dash-dot line) of the different channels of architecture A, at each $\mathrm{LPF}_{2}$ output, for $P_{\mathrm{p}}=15 \mathrm{dBm}, G_{\mathrm{e}}$ $=40 \mathrm{~dB}, G_{\mathrm{o} 1}=40 \mathrm{~dB}, G_{\mathrm{o} 2}=30 \mathrm{~dB}, G_{\mathrm{r}}=20 \mathrm{~dB}$ and $P_{\text {in }}=-65 \mathrm{dBm}$.
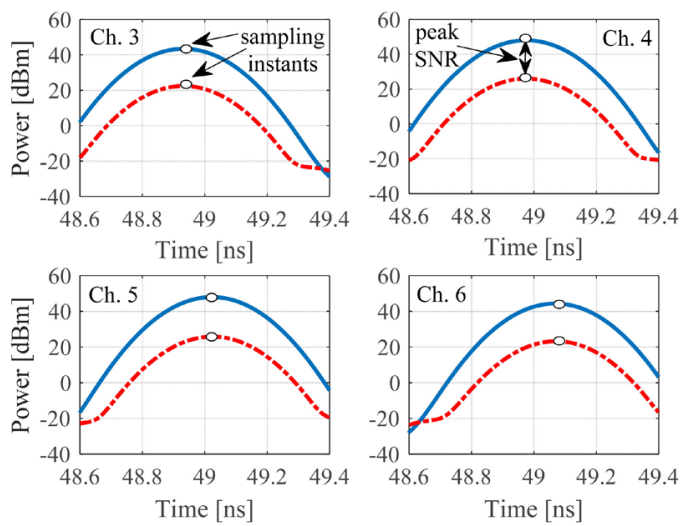

Figure 8. Instantaneous signal power (continuous line) and noise variance (dash-dot line) of the different channels of architecture B, at each $\mathrm{LPF}_{2}$ output, for $P_{\mathrm{p}}=15 \mathrm{dBm}, G_{\mathrm{e}}$ $=40 \mathrm{~dB}, G_{\mathrm{o} 1}=40 \mathrm{~dB}, G_{\mathrm{o} 2}=30 \mathrm{~dB}, G_{\mathrm{r}}=20 \mathrm{~dB}$ and $P_{\text {in }}=-65 \mathrm{dBm}$.

that, when compared with the curves of Figure 7, a residual power component is no longer present for the channels of interest. For architecture A, the residual pulses shown in Figure 5 corresponded to channels which also captured samples inside the radar pulse width. This is not the case for architecture B; the residual pulses in architecture B correspond to captured samples of channels 2 and 7, which are not shown in Figure 8, as these channels do not belong to the set of channels of interest (channels 3, 4, 5 and 6). Figure 8 also shows that the signal power and noise variance are confined in a time slot larger than the radar pulse width. This is attributed to decrease in bandwidth of the low-pass filter $\mathrm{LPF}_{2}$ for architecture $\mathrm{B}$, which results in an increase in time spreading, compared to the curves of Figure 7.

Figure 9 shows the peak SNR levels as a function of the channel index, for both architectures, for a peak power of the pulsed source of $15 \mathrm{dBm}$ and a radar received power of $-65 \mathrm{dBm}$ (distance of 7.6 meters). Additionally, $G_{\mathrm{e}}=40 \mathrm{~dB}$ and $G_{\mathrm{r}}=\{20,30,40\} \mathrm{dB}$ are considered. Only the results with $G_{\mathrm{o} 1}$ and $G_{\mathrm{o} 2}$ respecting the OA power limits and PIN-PD power limits are shown. Figure 9 


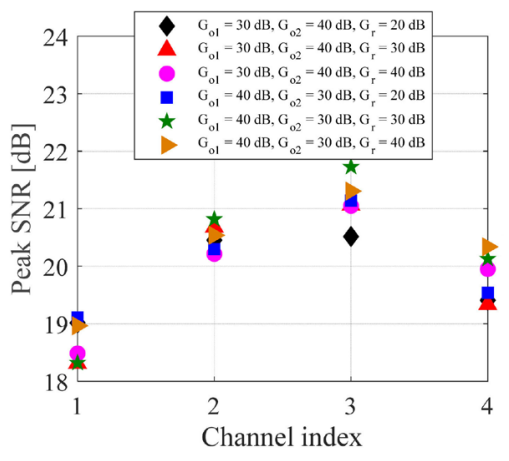

(a)

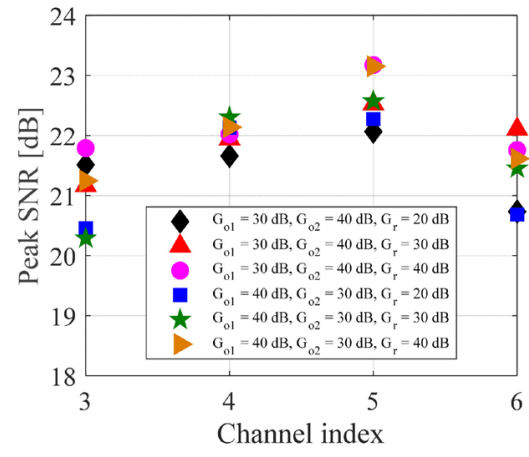

(b)

Figure 9. Peak SNR levels as a function of the channel index for a peak power of the pulsed source of $15 \mathrm{dBm}$ and a radar received power of $-65 \mathrm{dBm}$ (distance of 7.6 meters), for (a) architecture A and (b) architecture B. Additionally, $G_{\mathrm{e}}=40 \mathrm{~dB}$ and $G_{\mathrm{r}}=\{20,30$, $40\} \mathrm{dB}$ are considered. Only the results with $G_{\mathrm{o} 1}$ and $G_{02}$ respecting the OA power limits are shown.

shows that the channels with lower SNR are the first channel and third channels for architectures A and B, respectively. This is attributed mostly to the power attenuation induced by the pulsed source in these channels and reveals the importance of using optical pulsed sources with flattened shape. Figure 9 also shows that the peak SNR variation of the different channels does not exceed $4 \mathrm{~dB}$ and 3 $\mathrm{dB}$ for architectures A and B, respectively. The peak SNR variation is smaller for architecture $B$ due to the smaller difference in power loss induced by the pulsed source. In each channel of interest, for both architectures, the SNR variation does not exceed $1.5 \mathrm{~dB}$, for all considered gain combinations. Figure 9 also shows that, for all gain combinations, the peak SNRs are approximately $1.5 \mathrm{~dB}$ higher for architecture B, when comparing with architecture A. Since the non-flat amplitude response of the pulsed source induces less attenuation to the channels of interest of architecture $\mathrm{B}$, the signal power at $\mathrm{OA}_{2}$ output is higher for architecture $B$. This results in a small improvement in the overall SNR of the different channels of architecture B, when comparing with architecture A.

Since the main interest is to obtain the highest peak SNR for a specific distance, in this work we define the peak SNR of interest as the highest peak SNR of the most limiting channel. Hereafter, this is referred as the worst-case scenario. For architecture A, the worst-case scenario is the highest peak SNR of the first channel. For architecture B, the worst-case scenario can be the highest peak SNR of the third or the sixth channel, as they present similar SNR values for some gain combinations. In the following analysis, the third channel is chosen as the worst-case scenario for architecture B. Additionally, the best-case scenario is also considered, which corresponds to the highest SNR levels for the third channel for architecture A, and to the highest SNR levels for the fifth channel for architecture B.

Figure 10 shows the peak SNR levels, for the worst-case and best-case scenarios, as a function of the distance between the radar antenna and the sensing object, for peak powers of the pulsed source of 10,15 and $20 \mathrm{dBm}$, for both 


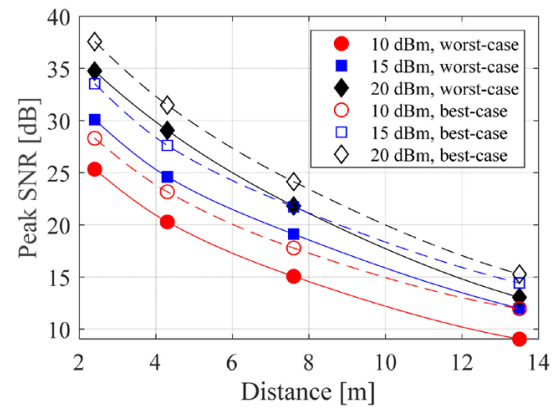

(a)

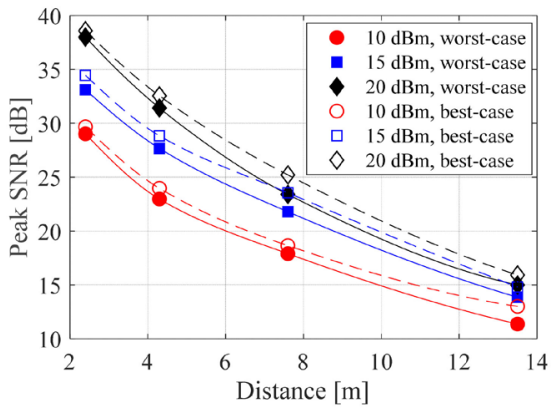

(b)

Figure 10. Peak SNR levels, for the worst-case and best-case scenarios, as a function of the distance between the radar antenna and the sensing object, for (a) architecture A and (b) architecture B. Results with peak powers of the pulsed source of 10, 15 and $20 \mathrm{dBm}$ are shown.

architectures. Figure 10 shows that the SNR decrease with increasing distance is stronger for higher peak powers of the pulsed source. For instance, for architecture A, with $P_{\mathrm{p}}=10 \mathrm{dBm}$, the SNR decreases approximately $16 \mathrm{~dB}$ when the distance increases from 2.4 to 13.5 meters. With $P_{\mathrm{p}}=20 \mathrm{dBm}$ and for the same distance increase, the SNR decreases approximately $22 \mathrm{~dB}$. Figure 10 also shows that, for a distance of 2.4 meters, peak SNR levels higher than $25 \mathrm{~dB}$ are achieved for both architectures. When the distance increases to 4.3 meters, peak SNRs higher than $20 \mathrm{~dB}$ are obtained. For a distance of 13.5 meters and for both scenarios, the peak SNR levels are substantially lower: for instance, the peak SNR for the worst-case scenario decreases to $9 \mathrm{~dB}$ for architecture $\mathrm{A}$ and to $11 \mathrm{~dB}$ for architecture B. Figure 10 also shows that the SNR variation between the worst-case and best-case scenarios, for the same peak power of the pulsed source and the same distance between the radar antenna and the sensing object, is higher for architecture A, when comparing with architecture B. This is a direct consequence of the non-flat amplitude response of the pulsed source, which induces higher power levels to the channels of interest of architecture B, when comparing with the power levels induced on the channels of architecture A. Figure 10 also shows that when comparing the worst-case and best-case scenarios for the considered peak powers of the pulsed source and for a distance of $2.4 \mathrm{~m}$, the peak SNR varies between 25 and $38 \mathrm{~dB}$ for architecture A and between 29 and 39 $\mathrm{dB}$ for architecture $\mathrm{B}$. When the distance increases to $13.5 \mathrm{~m}$, the peak SNR varies between 9 and $15 \mathrm{~dB}$ for architecture $A$ and between 11 and $16 \mathrm{~dB}$ for architecture B. In conclusion, architecture B provides higher peak SNR levels when compared with architecture A. Figure 10 also shows that the difference between both architectures does not exceed $4 \mathrm{~dB}$, when considering the same peak powers of the pulsed source and the same scenario.

\section{Conclusion}

The performance of the WDM Ph-ADC system with 4 and 8 channels (employing ADCs with sampling rate of 5 GSamples/s and 2.5 GSamples/s, respectively) 
used for the digitization of high-resolution radar signals has been evaluated numerically by using the peak SNR metric. Results show that, when comparing the worst-case and best-case scenarios for the considered peak powers of the pulsed source and for a distance of $2.4 \mathrm{~m}$, the peak SNR varies between 25 and $38 \mathrm{~dB}$ for architecture A and between 29 and $39 \mathrm{~dB}$ for architecture $\mathrm{B}$. When the distance increases to $13.5 \mathrm{~m}$, the peak SNR varies between 9 and $15 \mathrm{~dB}$ for architecture $A$ and between 11 and $16 \mathrm{~dB}$ for architecture B. In conclusion, architecture B provides higher peak SNR levels when compared with architecture $A$, and the peak SNR difference between both architectures does not exceed $4 \mathrm{~dB}$, when considering the same peak powers of the pulsed source and the same scenario. The SNR variation of both architectures can be reduced by choosing optical pulsed sources with more flattened spectrum or by leveling the different power levels induced by the optical pulsed source using optical attenuators for each channel.

\section{Acknowledgements}

This work was supported by the European Regional Development Fund through the Competitiveness and Internationalization Operational Program, Regional Operational Program of Lisbon, Regional Operational Program of the Algarve, in component FEDER, and the Foundation for Science and Technology (FCT), Project RETIOT, POCI-01-0145-FEDER-016432. This work was also supported by the FCT researcher contract IF/01225/2015/CP1310/CT0001.

\section{Conflicts of Interest}

The authors declare no conflicts of interest regarding the publication of this paper.

\section{References}

[1] Zhang, F., Guo, Q., Wang, Z.,Zhou, P., Zhang, G., Sun, J. and Pan S. (2017) Photonics-Based Broadband Radar for High-Resolution and Real-Time Inverse Synthetic Aperture Imaging. Optics Express, 25, 16274-16281.

https://doi.org/10.1364/OE.25.016274

[2] Yu, L., Zou, W., Yang, G., Li, X. and Chen, J. (2018) Switching Response of Dual-Output Mach-Zehnder Modulator in Channel-Interleaved Photonic Analog-to-Digital Converter. Chinese Optics Letters, 16, Article ID: 120602. https://doi.org/10.3788/COL201816.120602

[3] Cruz, P., Alves, T. and Cartaxo, A. (2018) Relaxing the ADC Sampling Rate in High-Resolution Radar Systems through Photonic Analogue-to-Digital Conversion. Proceedings of ICTON2018, Bucharest, 1-5 July 2018, 1. https://doi.org/10.1109/ICTON.2018.8473984

[4] Yang, J., Li, S., Xiao, X., Wu, D., Xue, X. and Zheng, X. (2018) Broadband Photonic ADC for Microwave Photonics-Based Radar Receiver. Chinese Optics Letters, 16, Article ID: 060605. https://doi.org/10.3788/COL201816.060605

[5] Mahjoubfar, A., Churkin, D., Barland, S., Broderick, N., Turitsyn, S. and Jalali, B. (2017) Time Stretch and Its Applications. Nature Photonics, 11, 341-351. https://doi.org/10.1038/nphoton.2017.76 
[6] Yang, G., Zou, W., Yu, L., Wu, K. and Chen, J. (2016) Compensation of Multi-Channel Mismatches in High-Speed High-Resolution Photonic Analog-to-Digital Converter. Optics Express, 24, 24061-24074. https://doi.org/10.1364/OE.24.024061

[7] Zhang, H., Zou, W., Yang, G. and Chen, J. (2016) Dual-Output Modulation in Time-Wavelength Interleaved Photonic Analog-to-Digital Converter Based on Actively Mode-Locked Laser. Chinese Optics Letters, 14, Article ID: 030602. https://doi.org/10.3788/COL201614.030602

[8] Khilo, A., Spector, S., Grein, M., Nejadmalayeri, A., Holzwarth, C., Sander, M., Dahlem, M., Peng, M., Geis, M., DiLello, N., Yoon, J.,Motamedi, A., Orcutt, J., Wang, J., Sorace-Agaskar, C., Popović, M., Sun, J., Zhou, G.-R., Byun, H., Chen, J., Hoyt, J., Smith, H., Ram, R., Perrott, M., Lyszczarz, T., Ippen, E. and Kärtner, F. (2012) Photonic ADC: Overcoming the Bottleneck of Electronic Jitter. Optics EXpress, 20, 4454-4469. https://doi.org/10.1364/OE.20.004454

[9] McKinney, J. (2014) Photonics Illuminates the Future of Radar. Nature, 507, 310-312. https://doi.org/10.1038/507310a

[10] Fard, A., Gupta, S. and Jalali, B. (2013) Photonic Time-Stretch Digitizer and Its Extension to Real-Time Spectroscopy and Imaging. Laser \& Photonics Reviews, 7, 207-263. https://doi.org/10.1002/lpor.201200015

[11] Callahan, P., Dennis, M. and Clark Jr., T. (2012) Photonic Analog-to-Digital Conversion. Johns Hopkins APL Technical Digest, 30, 280-286. https://www.jhuapl.edu/Content/techdigest/pdf/V30-N04/30-4-Callahan.pdf

[12] Yang, G., Zou, W., Yu, L. and Chen, J. (2018) Influence of the Sampling Clock Pulse Shape Mismatch on Channel-Interleaved Photonic Analog-to-Digital Conversion. Optics Letters, 43, 3530-3533. https://doi.org/10.1364/OL.43.003530

[13] Yang, G., Zou, W., Yuan, Y. and Chen, J. (2018) Wideband Signal Detection Based on High-Speed Photonic Analog-to-Digital Converter. Chinese Optics Letters, 16, Article ID: 030601. https://doi.org/10.3788/COL201816.030601

[14] Xu, Y., Li, S., Xue, X., Xiao, X., Zheng, X. and Zhou, B. (2019) An Interleaved Broadband Photonic ADC Immune to Channel Mismatches Capable for High-Speed Radar Imaging. IEEE Photonics Journal, 11, Article ID: 5502009. https://doi.org/10.1109/JPHOT.2019.2926399

[15] Alves, T. and Cartaxo, A. (2011) SNR Approach for Performance Evaluation of Time-Stretching Photonic Analogue to Digital Converter System. Optics Express, 19, 1493-1509. https://doi.org/10.1364/OE.19.001493

[16] Zhang, F., Guo, Q. and Pan, S. (2017) Photonics-Based Real-Time Ultra-HighRange-Resolution Radar with Broadband Signal Generation and Processing. Scientific Reports, 7, Article No. 13848. https://doi.org/10.1038/s41598-017-14306-y

[17] PriTel UOC Series. http://www.pritel.com/pdfs/UOC_1550nm.pdf

[18] Calmar PSL-10-TT. http://www.calmarlaser.com/docs/PSL_10G_pulsewidth.pdf 